\title{
Análisis del pensamiento algebraico desde la teoría cultural de la objetivación'
}

\section{Analysis of algebraic thinking from cultural theory of objectification \\ Análise do pensamento algébrico da teoria cultural de objetivação}

Recibido: mayo 2013

Aceptado: agosto 2013
John Gómez Triana ${ }^{2}$

Javier Mojica Vargas ${ }^{3}$

\section{Resumen}

Se presenta un taller práctico para docentes investigadores que tiene como objetivo proporcionar herramientas que permitan ampliar la mirada de los signos que dan cuenta del pensamiento algebraico en la resolución de tareas sobre generalización de patrones. Esta intención se capitaliza al proponer a los asistentes un ejercicio de análisis de la actividad matemática de un grupo de estudiantes cuando resuelven una Tarea sobre generalización de patrones de una secuencia figural. Para tal fin se usan algunos elementos de la teoría cultural de la objetivación como categorías de análisis.

Palabras clave: Aprendizaje; procesos cognitivos; generalización; pensamiento algebraico; semiótica cultural; medios semióticos de objetivación; profesor; formación continua de profesores.

\section{Abstract}

We present a workshop for teachers and researchers that aims to provide tools to expand the look of the signs that show the algebraic thinking in solving tasks on generalization of patterns. This intention is capitalized attendees to propose an exercise in mathematical activity analysis of a group of students when Task resolve a generalization of a sequence figural patterns. To this end, use some elements of the cultural theory of objectification as categories of analysis.

Keywords: learning, cognitive processes generalization algebraic thinking, cultural semiotics, semiotic means of objectification teacher, training of teachers.

\section{Resumo}

Nós apresentamos um workshop para professores e pesquisadores, que visa fornecer ferramentas para expandir o olhar dos sinais que mostram

Artículo de Investigación

2 InstitutoTécnico Industrial Piloto. Contacto: johngomezt@gmail.com

3 Universidad Distrital Francisco José de Caldas. Bogotá, Colombia. Contacto: javiermojicav@hotmail.com 
o pensamento algébrico na resolução de tarefas de generalização de padrões. Esta intenção é capitalizado participantes a propor um exercício de análise de um grupo de estudantes de atividade matemática quando Task resolver uma generalização de uma seqüência de padrões figurativos. Para este fim, utilizar alguns elementos da teoria cultural de objetivação como categorias de análise.

Palavras-chave: aprendizagem, processos cognitivos generalização pensamento algébrico, semiótica cultural, os meios semióticos de objetificação professor, a formação de professores.

\section{Presentación y marco de referencia conceptual}

Se presenta un taller práctico para docentes investigadores que tiene como objetivo proporcionar herramientas que permitan ampliar la mirada de los signos que dan cuenta del pensamiento algebraico en la resolución de tareas sobre generalización de patrones. Para ello nos situamos desde una perspectiva semiótico cultural de la enseñanza y el aprendizaje de las matemáticas planteada por Radford (2006a) en la teoría cultural de la objetivación (TCO). Esta teoría proporciona una serie de herramientas para analizar la actividad matemática de los estudiantes desde una perspectiva semiótica cultural. En este caso, tales herramientas son utilizadas en el análisis de la actividad matemática, de un grupo de estudiantes de grado décimo, cuando resuelven tareas asociadas a la generalización de patrones de secuencias figurales y numéricas que hicieron parte del proyecto de investigación presentado como tesis de maestría de Gómez (2013) ${ }^{4}$.

El taller aquí presentado toma un marco de referencia conceptual basado en la concepción de álgebra y pensamiento algebraico desde el enfoque semiótico-cultural propuesto por Radford (2006b, 2008, 2010a). En este enfoque se abordan planteamientos teóricos sobre una mirada no mentalista del pensamiento, se amplía la idea de signo, no solo como medio de representación de los objetos algebraicos, sino también como elemento constitutivo del pensamiento y de la actividad, introduciendo así el concepto de Medio Semiótico de Objetivación y teorizando la idea de Procesos de Objetivación. Además, en un contexto de generalización de patrones, se caracteriza el pensamiento algebraico a partir de la analiticidad, la indeterminancia y la designación simbólica. Se propone una tipología de formas del pensamiento algebraico basada en los medios semióticos de objetivación movilizados por los estudiantes cuando resuelven tareas de generalización de patrones en secuencias figurales. Con base en lo anterior a continuación se desarrollan los constructos teóricos de la TCO que serán utilizados como herramientas de análisis de las actividades diseñadas para el taller.

Inicialmente es importante mencionar que en la TCO se profundiza y se amplía la idea de signo desde la perspectiva de Vygotski. Los trabajos de Vygotski sugieren considerar que el signo desempeña una función mediadora entre el individuo y su contexto ${ }^{5}$, es decir, los signos son considerados como algo que abarca términos lingüísticos orales y escritos, símbolos matemáticos, gestos, etc. (Vergel, 2011). En este sentido, la noción de signo -derivada del trabajo de Vygotski - en Radford incluye gestos, artefactos, ritmo y actividad kinestésica. De acuerdo con Radford (2006a, 2006b, 2010a), los signos juegan un rol importante en tanto son los elementos que no sólo ayudan a realizar la actividad reflexiva, sino que hacen parte constitutiva de la actividad y de los procesos sociales; al decir de Vergel (2011), la actividad se hace con signos y se piensa con los signos.

4 En este trabajo se realizó un estudio de los medios semióticos de objetivación y procesos de objetivación desarrollados por un grupo de estudiantes de grado décimo de un colegio público de la ciudad de Bogotá-Colombia. Es así como los datos a utilizar, en el taller aquí presentado, hacen parte de la recolección de información realizada para el proyecto de investigación reportado en Gómez (2013).

5 El signo, además, permite ese pasaje entre lo interpsicológico y lo intrapsicológico que asegura la reconstrucción interna de la acción, esto es, de su internalización (Radford, 2006b) 
La idea de Medios Semióticos de Objetivación (artefactos, gestos, símbolos, palabras) es considerada como recursos semióticos. Para Radford (2010a, 2010b) los medios semióticos de objetivación no son únicamente herramientas por medio de las cuales manipulamos el mundo, sino mediadores de nuestros actos intencionales, portadores de una conciencia histórica construida a partir de la actividad cognitiva de las generaciones precedentes. De acuerdo con Radford (2010b), los medios semióticos de objetivación estratifican el objeto matemático en niveles de generalidad de acuerdo con la actividad reflexiva que ellos median.

Por otro lado, en el marco de la TCO se estudian los procesos sociales por medio de los cuales los sujetos aprenden a pensar de acuerdo a modos culturales ya establecidos. Dichos procesos es lo que Radford (2010b) llama procesos de objetivación. En este contexto, interesa mostrar y replicar el análisis del desarrollo conceptual (desarrollo de pensamiento algebraico) a través de la implementación y el análisis de tareas asociadas a la generalización de patrones en secuencias figurales utilizadas en la tesis de maestría de Gómez (2013). Dichas tareas fueron propuestas con la intención de producir un desarrollo en los estudiantes, visto como un proceso social de aproximación de significados subjetivos o personales a los significados histórico-culturales plasmados en la semiótica algebraica. En Radford ${ }^{6}(2008)$ se distinguen dos de estos procesos que el autor llama Iconicidad y Contracción Semiótica. El primero de ellos es el proceso mediante el cual los estudiantes se basan en experiencias anteriores para orientarse en una nueva situación; el segundo lleva al individuo a hacer una elección entre lo que se considera relevante o irrelevante ${ }^{7}$.

Es así como en la TCO se busca llamar la atención sobre el hecho de que el lenguaje simbólico del álgebra no es la única herramienta semiótica que da cuenta del pensamiento algebraico. Como señala Mason, citado en Radford (2010a), "la manipulación de los símbolos es sólo una pequeña parte de lo que el álgebra es en realidad". Lo que se propone entonces es que el álgebra aborda la indeterminancia tratada analíticamente, pero en lugar de conceder al simbolismo alfanumérico el derecho exclusivo para designar y expresar la indeterminación, se afirma que se trata de sólo una de las varias formas semióticas equipadas para lograrlo.

Finalmente, teniendo en cuenta que las tareas utilizadas para el presente taller se encuentran inmersas en un contexto de generalización de patrones, se enmarcan en el pensamiento algebraico y constituyen una vía para su desarrollo, se hace necesario mencionar que en Radford (2010b) se propone una tipología de formas del pensamiento algebraico determinadas por los medios semióticos de objetivación movilizados, tal tipología se describe a continuación:

- El Pensamiento Algebraico Factual. Aquí la indeterminación queda implícita en palabras y gestos y el ritmo constituye la sustancia de la semiótica en los estudiantes en un proceso llamado fórmulas en acción.

- El Pensamiento Algebraico Contextual. Aquí la indeterminación se convierte en un objeto explícito del discurso. Los gestos y ritmos son reemplazados por deícticos lingüísticos, adverbios, etc.

- El Pensamiento Algebraico Simbólico. Aquí las fórmulas en lugar de ser un dispositivo de resumen de cálculos aparecen como narraciones vividas; son íconos que ofrecen una especie de descripción espacial de la figura y acciones que se llevarán a cabo.

Es así como la idea de signo, el concepto de medio semiótico de objetivación, el constructo teórico de proceso de objetivación y las formas de pensamiento algebraico constituyen los elementos teóricos de

6 Una de las tesis fundamentales en la propuesta teórica de Vygotski es que la instrucción precede al desarrollo, lo cual sugiere que el pensamiento se puede desarrollar.

7 D’Amore (2006) tematiza la contracción semiótica en términos de la economía de los recursos semióticos usados, lo cual se traduce en una sobriedad a quien piensa y se expresa. 
análisis de la actividad matemática de los estudiantes con los cuales los asistentes al taller realizarán el análisis de la Tarea propuesta.

\section{Descripción de las actividades}

El taller se desarrolla en dos sesiones de 90 minutos cada una, las cuales está distribuidas en 7 momentos ( 3 momentos en la primera sesión y 4 en la segunda). Como ya se mencionó, el taller tiene como objetivo realizar un ejercicio de análisis de la actividad matemática de un grupo de estudiantes cuando resuelve una Tarea sobre generalización de patrones de una secuencia figural. Para tal fin se usarán los elementos teóricos de la TCO expuestos en el marco de referencia. A continuación se describe cada uno de los momentos en los que se divide el taller

Sesión 1. Momento 1: Resolución de la tarea 1. Se conforman grupos de tres asistentes de los cuales dos de ellos darán solución a la Tarea 1 (Ver Figura 1). El tercer integrante asumirá el rol de observador recopilando en un instrumento de recolección de información (Ver Figura 2) la forma de proceder y las acciones realizadas por los dos asistentes resolutores durante la solución de la Tarea 1.

Momento 2: Presentación del video 1. Se presenta el video de la actividad matemática realizada por estudiantes de grado décimo al resolver la tarea 1. Se entregan las transcripciones correspondientes al video 1. Posteriormente se plantea la discusión acerca de lo que puede decirse sobre la forma de proceder de los estudiantes.
Momento 3: Análisis del video 1 Los talleristas presentan el análisis multimodal ${ }^{\underline{8}}$ realizado a la actividad matemática de los estudiantes del video 1 cuando resuelven la tarea 1 utilizando como herramientas de análisis los constructos teóricos de la TCO.

Sesión 2. Momento 4: Resolución de la tarea 2. Los grupos de asistentes resuelven la tarea 2 (Ver figura 3).

Momento 5: Presentación videos 2 y 3. Correspondientes a la actividad matemática de estudiantes de grado décimo al resolver la tarea 2, se entregan las transcripciones de los respectivos videos.

Momento 6. Ejercicio de análisis de los videos 2 y 3. Teniendo en cuenta las unidades de análisis (medios semióticos de objetivación, procesos de objetivación, formas de pensamiento algebraico) presentadas y discutidas en el video 1 , se solicita a los asistentes asumir el rol de investigadores e inferir cuáles serían los medios semióticos y los procesos de objetivación presentes en los videos proyectados.

Momento 7: Discusión y reflexión. Finalmente se pretende plantear una reflexión frente a la naturaleza y las formas del pensamiento algebraico desde una perspectiva semiótico cultural, a partir de los hallazgos del ejercicio de análisis realizado, a la luz de los elementos teóricos de la TCO y comparado con las vivencias de los asistentes en la enseñanza o tratamiento de situaciones de generalización de patrones.

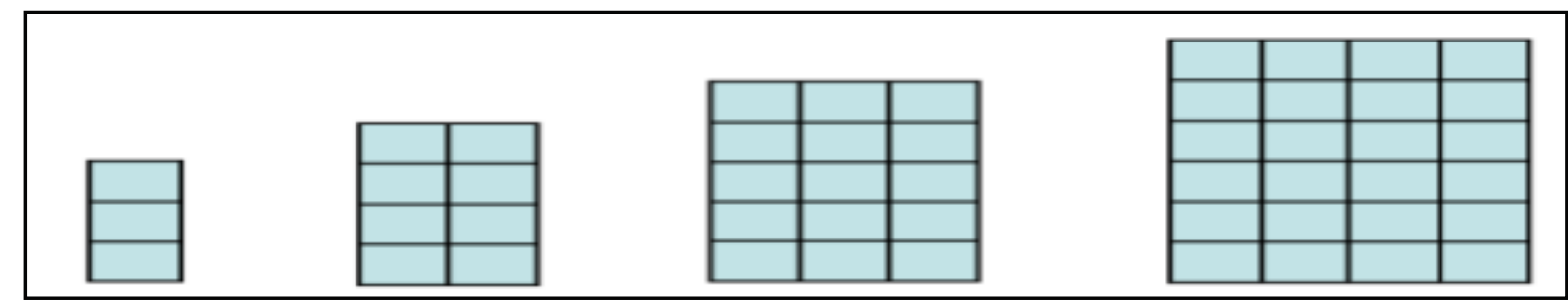

Fuente: Elaboración propia

8 En este tipo de análisis, se considera que lo gestuado, lo hablado y lo escrito por los estudiantes se analiza de manera conjunta, es decir, interesa centrar la mirada en la relación de los distintos sistemas semióticos activados en la actividad matemática. 


\section{Conclusiones}

Teniendo en cuenta que se trata de un taller teórico práctico las conclusiones obedecen a las reflexiones que la actividad realizada pueda provocar. Se espera entonces sensibilizar a los asistentes, en primer lugar, acerca de la necesidad de reconocer una serie de medios semióticos de objetivación y procesos de objetivación que caracterizan las formas de pensamiento algebraico que se manifiestan en una Tarea como la presentada en este escrito. En segundo lugar, interesa llamar la atención sobre la existencia de recursos semióticos que dan cuenta del pensamiento algebraico y que son diferentes al lenguaje alfanumérico que usualmente e histórico-culturalmente ha sido aceptado como la principal herramienta semiótica del álgebra.

\section{Referencias}

D'Amore, B. (2006). Objetos, significados, representaciones semióticas y sentido. En: Radford,L., D'Amore, B. (eds.) (2006). Semiotics, Culture and Mathematical Thinking. Número special de lla Rivista Relime (Cinvestav, México D.F., México). 177-196

Gómez, J. (2013). La generalización de patrones de secuencias figurales y numéricas:Un estudio de los medios semióticos de objetivación y procesos de objetivación en estudiantes de grado décimo. Tesis de maestría no publicada. Universidad Pedagógica Nacional. Bogotá-Colombia

Radford, L. (2006a). Elementos de una teoría cultural de la objetivación. Revista Latinoamericana de Investigación en Matemática Educativa, número especial sobre semiótica, cultura $\mathrm{y}$ pensamiento matemático (editores invitados: L. Radford y B. D’Amore), pp. 267-299.

Radford, L (2006b). Algebraic Thinking and the Generalization of Patterns: A semiotic perspective, PME-NA, Vol 1, pp. 2_21.

Radford, L. (2008). Iconicity and contraction: a semiotic investigation of forms of algebraic generalizations of patterns in different contexts. ZDM Mathematics Education, 40, 83-96.

Radford, L. (2010a). Algebraic thinking from a cultural semiotic perspective. Research in Mathematics Education, 12(1),1-19.

Radford, L. (2010b). Layers of generality and types of generalization in pattern activities. PNA, $4(2), 37-62$.

Radford, L. (2010c). Elementary Forms of Algebraic Thinking in Young Students. In M. Pinto.\& T. F. Kawasaki (Eds.). Proceedings of the 34th Conference of the International Group for the Psychology of Mathematics Education, Vol. 4, pp. 73-80. Belo Horizonte,Brazil: PME

Vergel, R. (2011). El signo en Vygotski y su vínculo con el desarrollo de los procesos psicológicos superiores. Ensayo no publicado presentado en el marco del seminario doctoral "Sujeto y Alteridad en el Discurso Pedagógico". Doctorado Interinstitucional en Educación, Universidad Pedagógica Nacional. Bogotá-Colombia. 